\title{
Gabor Filter Based Feature Vector for Dynamic Signature Recognition
}

\author{
H B Kekre \\ Sr. Professor, Computer Science Department, MPSTME \\ NMIMS University \\ Mumbai, Maharashtra, India
}

\author{
V A Bharadi \\ PhD Research Scholar, MPSTME \\ NMIMS University \\ Mumbai, Maharashtra, India
}

\begin{abstract}
Dynamic signature recognition is one of the commonly used biometric traits. In this paper we propose use of Gabor filters based feature for verification of dynamic signature. We incorporate the timing information available in the signature along with the Gabor filter response to generate the feature vector. Gabor filters have been widely used for image, texture analysis. Here we present analysis for the Gabor filter based feature vector of a dynamic signature.
\end{abstract}

\section{Categories and Subject Descriptors}

I.4.7 Image Processing and Computer vision

\section{General Terms}

Biometrics, Pattern Recognition, Image Processing.

\section{Keywords}

Dynamic Signature Recognition, Gabor Filter.

\section{INTRODUCTION}

With the advancement in the technology various methods of person identification are possible because of availability of affordable and co-operative sensors as well as capable processing computers and storage media. Another driving force behind widespread use of biometrics is the terrorism and need of better mechanism to protect sensitive areas where public interaction is more, like airports and railway stations. The methods are numerous, and are based on different personal characteristics. Voice, lip movements, hand geometry, face, odor, gait, iris, retina, fingerprint are the most commonly used authentication methods. All of these and behavioral characteristics are called biometrics [1][2][5][16].

The biometrics is most commonly defined as measurable psychological or behavioral characteristic of the individual that can be used in personal identification and verification. The biometrics is mainly concerned with 'what you are' rather than 'what you carry'. The driving force of the progress in this field is, above all, the growing role of the Internet and the requirements of society. Therefore, considerable implementations can be found in the area of electronic commerce and electronic banking systems and security applications of vital installations.

The biometrics has a significant advantage over traditional authentication techniques (namely smartcards, PIN numbers, passwords etc.) due to the fact that biometric characteristics of a person are not easily transferable, are unique of every person, and cannot be lost, stolen or broken. The choice of one of the biometric solutions depends on several factors [2]:

- User acceptance

- Level of security required

- Accuracy

- Cost and implementation time

Biometric and biomedical informatics are the fast developing scientific direction, studying the processes of creation, transmission, reception, storage, processing, displaying and interpretation of information in all the channels of functional and signal systems of living objects which are known to biological and medical science and practice.

\subsection{Handwritten Signature Recognition}

Handwritten signatures are one of the oldest biometrics traits. Its many applications include banking, credit card validation, security systems etc. Handwritten signature verification is divided in to two types - on-line verification and off-line verification [3][4][5]. On-line verification requires a stylus and an electronic digitizer tablet connected to a processing computer to grab dynamic signature information [5]. Off-line verification, on the other hand, deals the morphological or the external information contained in the signature. We can apply various Image and Signal processing based techniques to analyze there signatures.

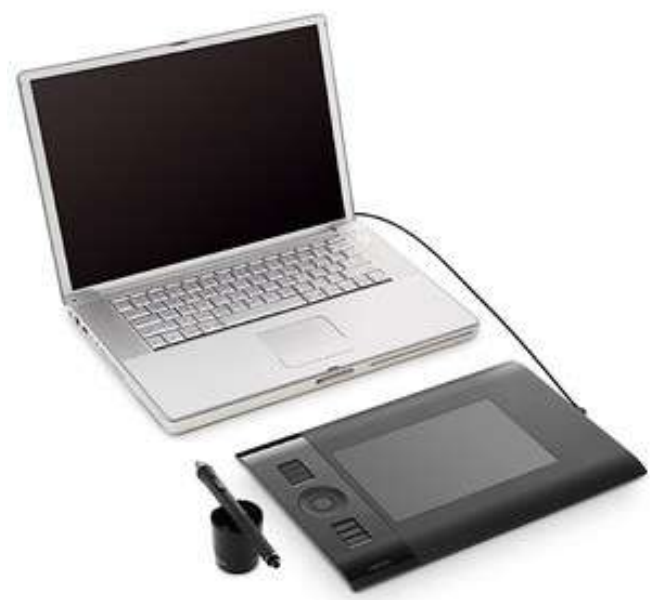

Figure 1. Capturing On-line Signature using Digitizer Tablet (Wacom Intuos4) 
In off-line signature recognition we are having the signature template coming from an imaging device like scanner, hence we have only static characteristic of the signatures. The person need not be present at the time of verification. Hence off-line signature verification is convenient in various situations like document verification, banking transactions etc. [1][3][7][8]. As we have a limited set of features for verification purpose, off-line signature recognition systems need to be designed very carefully to achieve the desired accuracy.

In On-line approach we can capture more information about the signature which contains the dynamic properties of signature[16][18]. We can find information about the writing speed, pressure points, strokes, acceleration as well as the static characteristics of signatures [6][16][18]. This leads to better accuracy because the dynamic characteristics are very difficult to imitate, but the system requires user co-operation and complex hardware. Digitizer tablets or pressure sensitive pads are used to scan signature dynamically, one such tablet is shown in Figure 1. The set of dynamic features from a dynamic signature is shown in Figure 2.
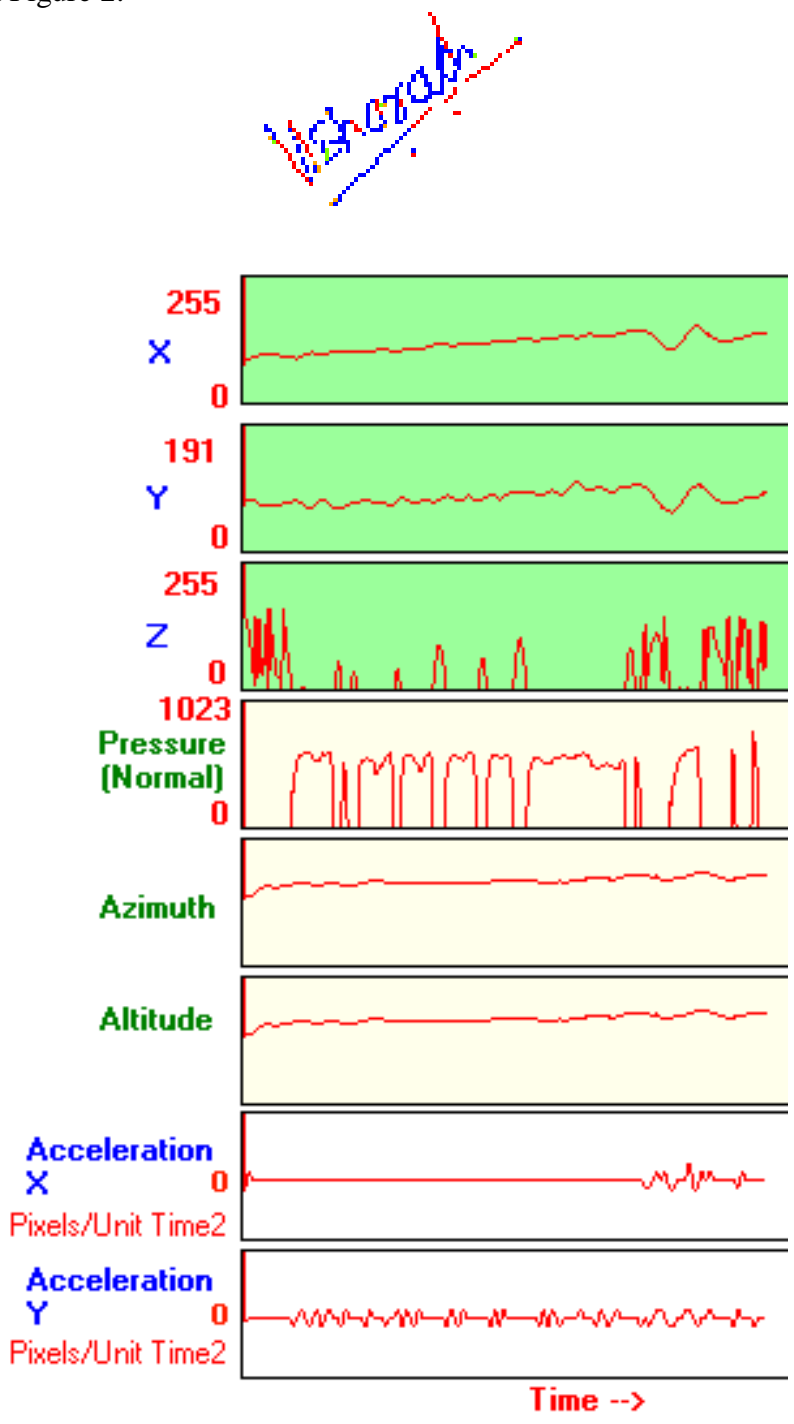

Figure2. Dynamic Features captured for a signature.

\section{ONLINE SIGNATURE RECOGNITION METHODS}

On-line signature recognition considers the dynamic characteristics of signatures. In [2] Jain \& Ross have used critical points, speed curvature angle as features and they have reported FRR $2.8 \%$ and FAR $1.6 \%$. They used common as well as writer dependent thresholds but it was observed that the writer dependent thresholds give better accuracy.

Considering another approach Lei, Palla and Govindarajalu [11] have proposed a technique for finding correlation between two signature sequences for online recognition, they mapped the occurrence of different critical points on signature and the time scale and the correlation between these sequences was evaluated using a new parameter called Extended Regression Square (ER2) coefficient the results were compared with an existing technique based on Dynamic Time Wrapping (DTW). They reported Equal Error rate (EER) $7.2 \%$ where the EER reported by DTW was $20.9 \%$ with user dependent thresholds.

In [12] Rhee and Cho used Model guided segmentation approach for segment-to-segment comparison to obtain consistent segmentation. They used discriminative feature selection for skilled as well as random forgeries. They reported EER $3.4 \%$. Nalwa [13] used a moment and torque base approach for on-line signature recognition. His work is based parameterizing each online curve over its normalized arc-length. These parameters are then represented along the length of the curve, in a moving coordinate frame. The measures of the curve within a sliding window that are analogous to the position of the center of mass, the torque exerted by a force, and the moments of inertia of a mass distribution about its center of mass. Further, He suggested the weighted and biased harmonic mean as a graceful mechanism of combining errors from multiple models of which at least one model is applicable but not necessarily more than one model is applicable. He recommended that each signature be represented by multiple models, these models, perhaps, local and global, shape based and dynamics based. The reported FRR was $7 \%$ and FAR was $1 \%$.

Authors also have proposed a dynamic signature recognition based system based on Vector Quantization based feature extraction. We have used Kekre's Median Codebook Generation Algorithm (KMCG)[16] on the time axis of the captured signature points [18]. Achieved Equal Error rate (EER) is at 94.08\% for TAR-TRR. and for False Acceptance \& rejection Rate (FAR-FRR)[1] above mention system has 5.92\% EER.

One thing that should be noted is that all these approaches need signature data with dynamic information. When the data comes from the hardware it is raw and we have to pre-process it to normalize the errors due to sampling, quantization, speed of hardware, signing position etc. We are using Wacom Intuos 4 for our experiments and we have also experienced the need of preprocessing the data. Doroz and Wrobel [14] have discussed this issue and proposed a technique of sampling the point uniformly to have equal number of points per unit time. They have used Signature verification Competition database [15]. Here we are using a signature pre-processing method based on Modified 
Dynamic Digital Analyzer Algorithm (MDDA) [18]. This gives better reconstruction of captured data. In this paper we are using Gabor Filter for feature extraction. In the next section this is discussed in detail.

\section{DATA ACQUISITION}

In order to capture signature features in dynamic mode we have to use digitizer tablets. In our experiment we have used a newly introduced device Wacom Intuos4. This digitizer is mainly used by graphics designers but have used it to capture dynamic signature of a user. For interfacing we have used WinTAB compatible VBTABLET ActiveX component [24]

A specialty of this device is that along with conventional parameters this device also gives $\mathrm{Z}$-co-ordinate of the tip of the pen while signing; this enables us to capture $\mathrm{X}, \mathrm{Y}, \mathrm{Z}$ co-ordinates of the signature in a 3 Dimensional space. Hence we say that is extracts multidimensional features of a signature. Note that the Z-Coordinate is sensed in a limit up to $2 \mathrm{~cm}$ (approx) from the scanner surface; but it is sufficient to detect the co-ordinates of tip when user lifts the pen while signing.

Typical features of the tablets is as follows

1. Active Area (W x D) 157.5 x $98.4 \mathrm{~mm}$

2. Connectivity-USB connectivity

3. Pressure levels -2048

4. Sensor pen without battery

5. Minimum ON weight (Minimum weight sensed by

the pen tip) - 1Gram.

6. Report rate- 197 Points per second

7. LPI - lines per inch-5080 lpi

Each captured points has multi-dimensional information. Each point contains information about X, Y, Z-Coordinates, Pressure, Azimuth and Altitude of the pen tip. Hence $i^{\text {th }}$ point $P_{i}$ can be considered as:

$$
p_{i}=\left\{x_{i}, y_{i}, z_{i}, p_{i}, A z_{i}, A l_{i}\right\}
$$

The captures points are pre-processed [18], to generate the final template, some of the captured signatures pressure information map is shown below. Different colors indicate different pressure levels.
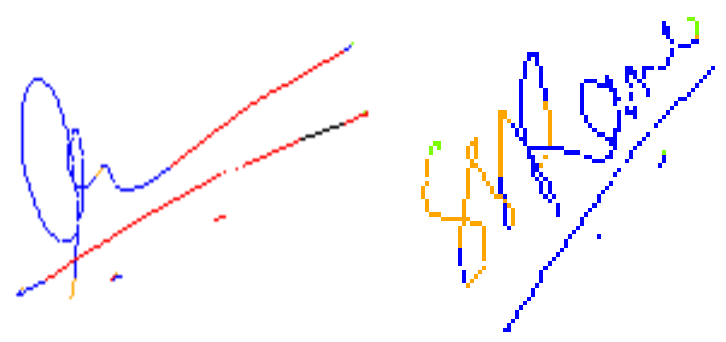

Figure 3. Dynamic Signature Pressure Maps Showing Different Pressure levels

The signature template thus generated is used for the feature extraction process. This is discussed in next section.

\section{GABOR FILTER}

Gabor filters are band-pass filters which have both orientationselective and frequency-selective properties and have optimal joint resolution in both spatial and frequency domains [17][19][20][21]. By applying properly tuned Gabor filters to a signature image, the texture information can be generated. These accentuated texture information can be used to generate feature vector. An even symmetric Gabor filter has the following general form in the spatial domain [17].

$\mathrm{h}(\mathrm{x}, \mathrm{y}, \theta, \mathrm{f})=\exp \left\{-\frac{1}{2}\left[\frac{x_{\theta}^{2}}{\sigma_{x}^{2}}+\frac{y_{\theta}^{2}}{\sigma_{y}^{2}}\right]\right\} \cos \left(2 \pi f x_{\theta}\right)$

Where $x_{\theta}=x \cos \theta+y \sin \theta$, and

$$
y_{\theta}=-x \sin \theta+y \cos \theta
$$

This filter consist of a Gaussian envelope (of parameters $\sigma \mathrm{x}$ and $\sigma y)$ modulated by a sinusoid of frequency $f$ along the direction of the $\mathrm{x} \theta$ axis. For our analysis we have used $(\sigma x=\sigma y=50$ Pixels) This is average width of the captured signature. The angle $\theta$ allows rotating the direction of the response. Example of 2D Gabor filter is shown in Figure 4.

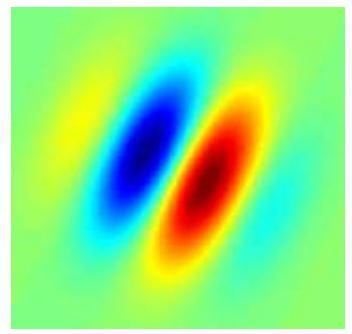

Figure 4. 2D Gabor Filter Response in Spatial Domain

Gabor filter has been used successfully in segmentation of fingerprint and palm prints [21][22][23], as well as their identification. Here we use this filter to generate the feature vector of dynamic signatures pressure map. We set the frequency by empirical study, the frequency $f$ is set as $7 \mathrm{pixel} /$ cycle $(1 / 7)$. The value of $\theta$ is given by

$$
\theta_{k}=\frac{\pi(k-1)}{m}
$$

(3)

$\mathrm{k}=1 \ldots \mathrm{m}$,

Where $\mathrm{m}$ denotes the number of orientations $(\mathrm{m}=8$ in this paper). For each image of size $\mathrm{M} \times \mathrm{N}$ centered at $(X, Y)$, with $\mathrm{W}$ even, we extract the Gabor Magnitude [22] as follows for $\mathrm{k}=1$, . $\ldots, \mathrm{m}$ :

$$
\begin{aligned}
& g\left(X, Y, \theta_{k}, f, \sigma_{x}, \sigma_{y}\right)= \\
& \left|\sum_{x_{0}=-M / 2}^{(M / 2)-1} \sum_{y_{0}=-N / 2}^{(N / 2)-1} I\left(X+x_{0}, Y+y_{0}\right) h\left(x_{0}, y_{0}, \theta_{k}, f, \sigma_{x}, \sigma_{y}\right)\right|
\end{aligned}
$$

Where $\mathrm{I}(\mathrm{x}, \mathrm{y})$ denotes the pressure level of the pixel $(\mathrm{x}, \mathrm{y})$. As a result, we obtain $\mathrm{m}$ Gabor features for each $\mathrm{M} \times \mathrm{N}$ size of the 
image( in our case we consider 256 X 192 Pixels Size signature template).

\subsection{Feature Vector Generation}

The feature vector represents the matching template, we actually find distance between the feature vector which contained extracted information. We have discussed the Gabor filter in the above section. Now we discuss how to use it to generate the feature vector.

In [25] A K jain et.al have used Gabor filter response to generate feature vector for fingerprint images. We use the technique in[25] on our pressure map of the signature and later incorporate the timing information with it. We use the tessellation with center aligning with the center of mass of the signature. The signature area is divided into total 48 sectors

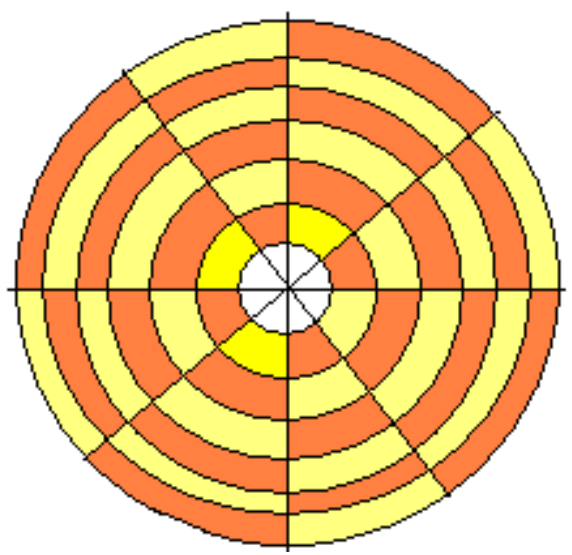

(a)

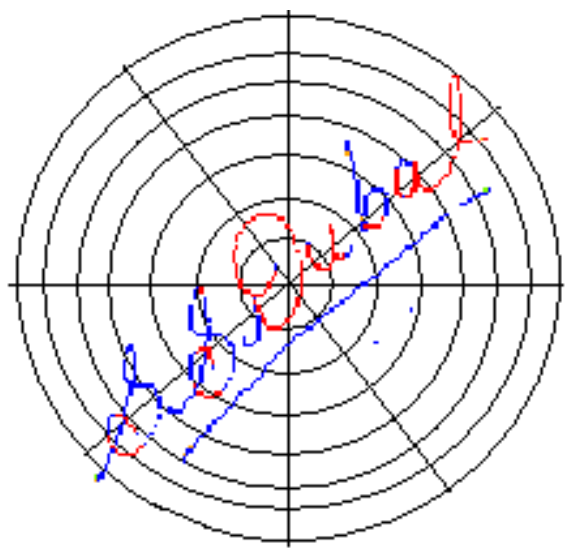

(b)

Figure 5. (a) 48 Sectors Tessellation Map orientation (b) Tessellation put over the signature for Gabor Filter based feature extraction

- Let $I(x, y)$ denote the gray level at pixel $(x, y)$ in an $M . \mathrm{X} N$ fingerprint image and let $(X c, \mathrm{Yc})$ denote the center point. The spatial tessellation of the signature space which consists of the region of interest is defined by a collection of sectors $\mathrm{Si}$, where the $i$ th sector $S i$ is computed in terms of parameters $(r, \mathrm{q})$ as follows[25]:

$$
\begin{aligned}
& S_{i}=\left\{(x, y) \mid b\left(T_{i}+1\right) . r<b\left(T_{i}+2\right), \theta_{i}<=\theta<=\theta_{i+1},\right. \\
& 1<=x<=N, 1<=y<=M\} \text {, } \\
& \text { where ( } b=10 \text { Pixels, } K=8 \text { Sectors in each band) and } \\
& T_{i}=i \operatorname{div} k \\
& \theta_{i}=(i \bmod k)\left(\frac{2 \pi}{k}\right) \\
& r=\sqrt{x-x_{c}^{2}+y-y_{c}^{2}} \\
& \theta=\tan ^{-1}\left(\frac{y-y_{c}}{x-x_{c}}\right)
\end{aligned}
$$

When we apply Gabor filter on the Signature pressure map, we have total 8 Planes of Gabor response corresponding to 8 Angles $(\mathrm{K}=8)$. Now using the above mentioned tessellation we calculate the mean and standard deviation of the Gabor response of the pixels of each sector (S0 to S47), and the we calculate Standard Deviation of Gabor response for each sector. In this way we get a 48 values of Gabor SD for one plane, such 8 planes are there hence the feature vector contains $48 \times 8$ Elements.

\subsubsection{Adding Timing Information to the Tessellation map}

To add dynamic property to the Gabor Tessellation, we generate the timing information about each sector. We have time stamp for each pixel when captured. In each sector we find pixel with lowest time stamp and assign that timestamp to the sector. If any sector does not have pixels then the time stamp holds infinite value $(\infty)$. Indicating omission of the sector. While evaluating Euclidian distance the sector values are sorted as per the timestamp and distance is calculated. The Gabor Response and corresponding Feature vector is shown in Figure 6.

\section{RESULTS}

The signatures are verified by evaluating the Euclidian distance between the feature vectors of signature templates. We have implemented this method in MS Visual C\# 2005 (.NET Fremework 2.0). On a AMD Athlon 64 processor, $1.8 \mathrm{GHz}$ and 1.5 GB RAM running Windows XP SP3.

For testing we have collected 250 signatures from 25 different users in average two sittings with time difference of one to two weeks. Volunteers were asked to forge Signatures. The result analysis is presented here.

We have tested to options (1) Gabor Filter based feature vector without Timestamp and (2) Gabor Filter based feature vector with Timestamp, We have used Euclidian distance between two vectors, currently we are evaluating the performance of the feature vector hence no training mechanism is used. Intra class as well as inter class testing is performed on the signature database.

Total 493 tests were performed and the results are summarized in Fig. 7 and Fig. 8. We have plotted True Acceptance Ratio (TAR) Vs True Rejection Ratio (TRR) and False Acceptance Ratio (FAR) Vs False Rejection Ratio (FRR) [1][2][3][21][22] for the analysis. 
0

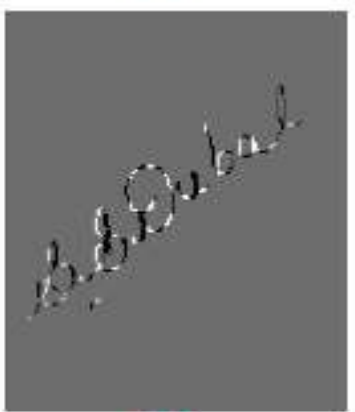

45
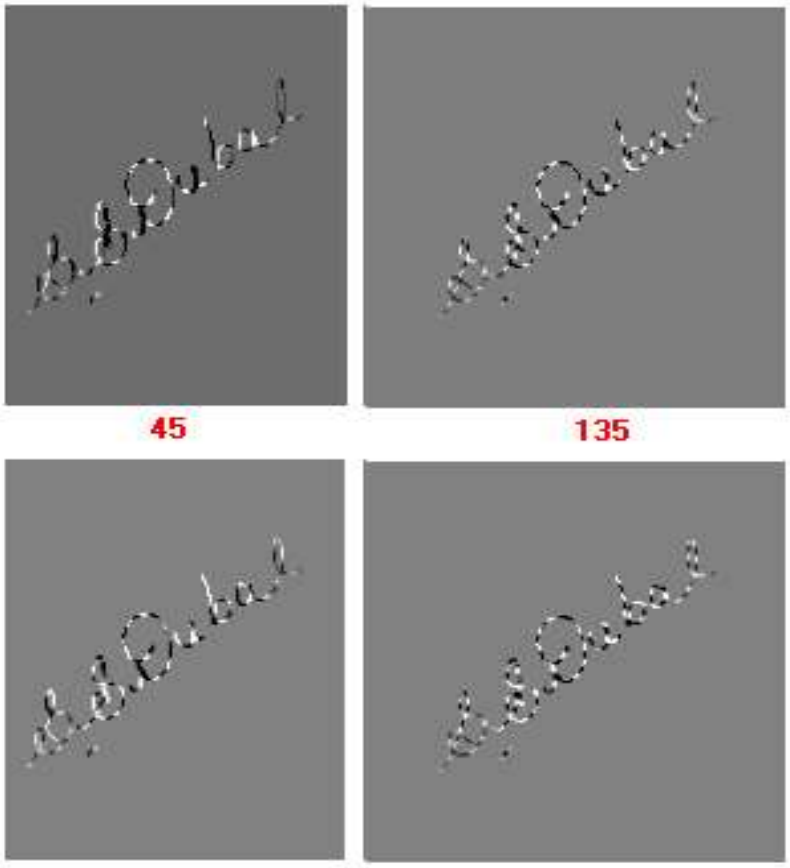

135
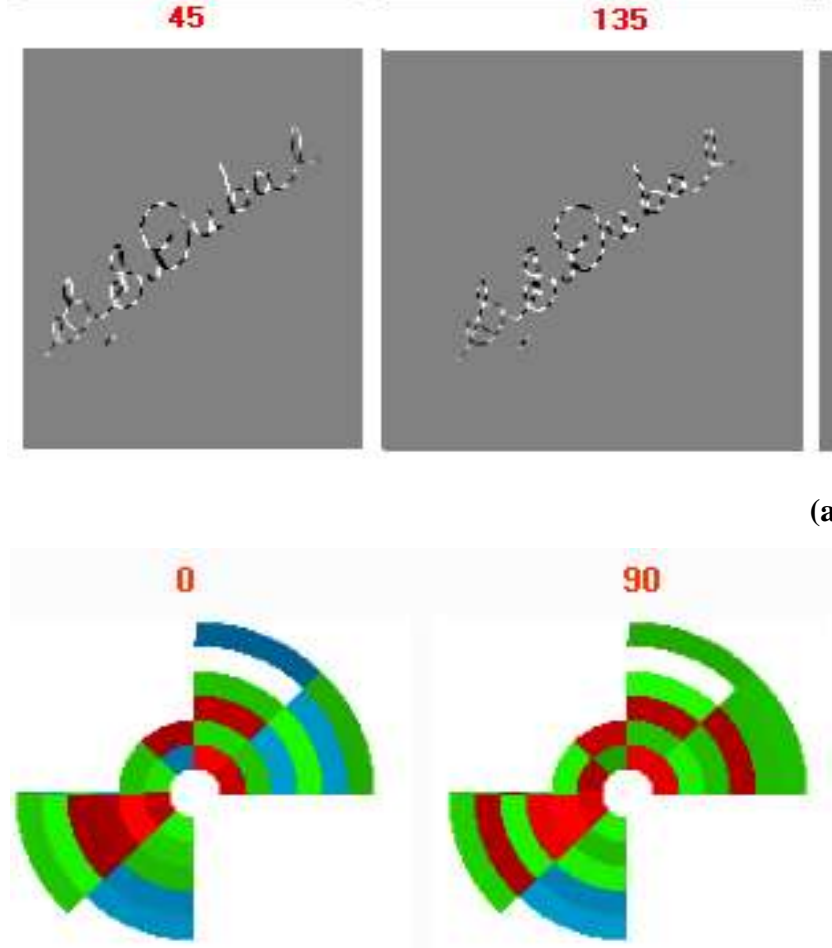

45

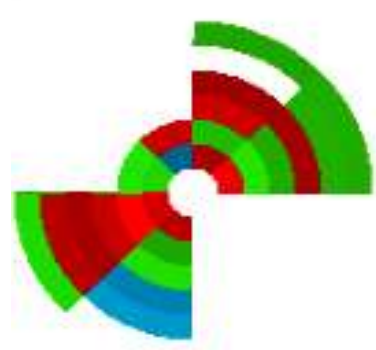

(a)

180

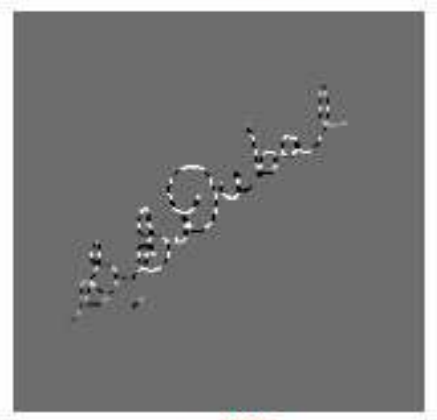

225

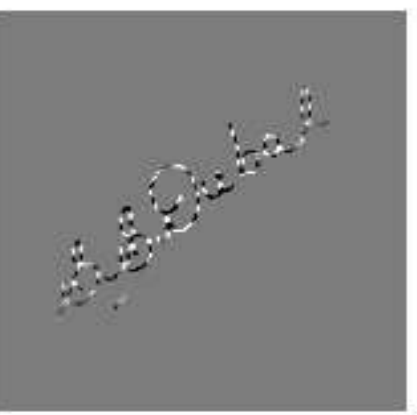

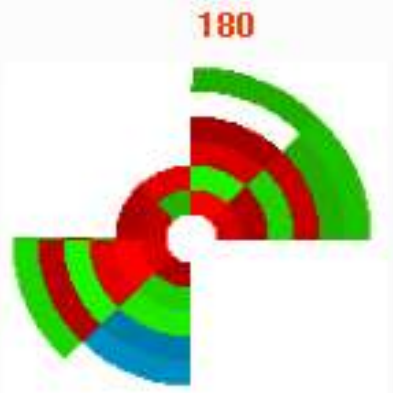

225

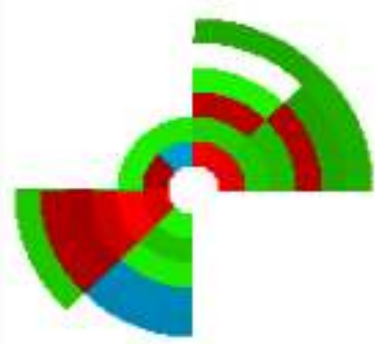

270

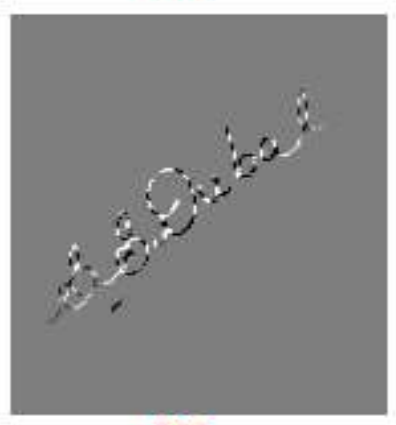

315

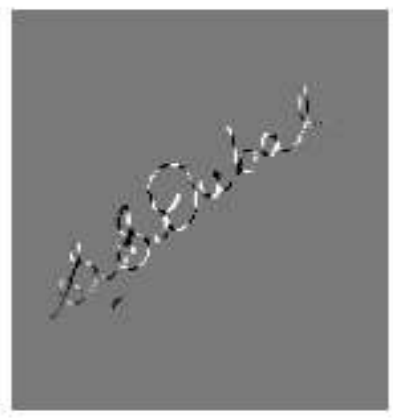

270

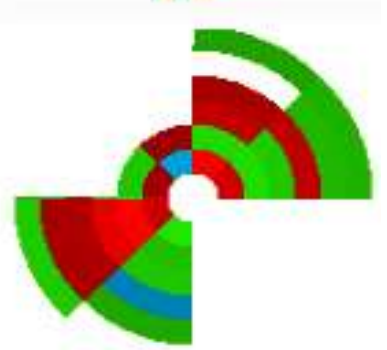

315

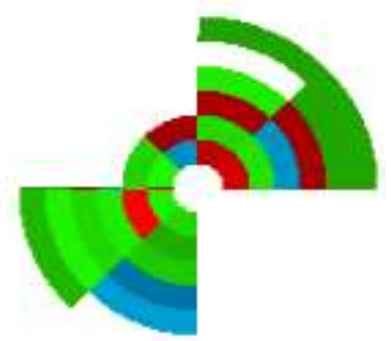

(b)

Figure 6. (a) Gabor Response of a Signature for 8 Angles as shown on top of each image (b) Corresponding Gabor Feature Vector, Blue color indicates low values, Green and Red indicate increasing Standard Deviation of Gabor Filter Response 


\subsection{Gabor Filter based Feature Vector without Timestamp}

The analysis shows that the Equal Error Rate (EER) [1][2][3] for Gabor Filter based Feature vector based classification is $90 \%$ for TAR Vs TRR plot, and it is $10 \%$ for FAR Vs FRR plot as shown in Fig 7(a) \& (b).

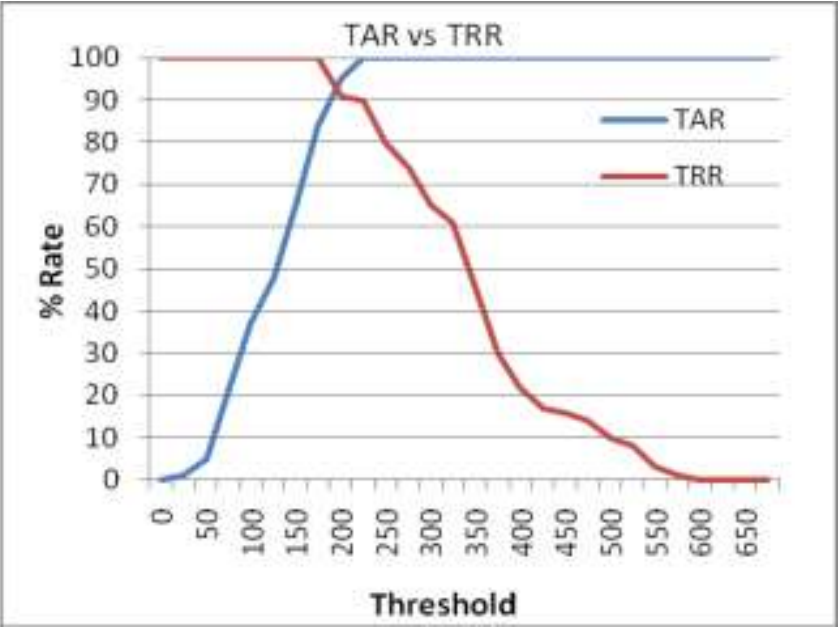

(a)

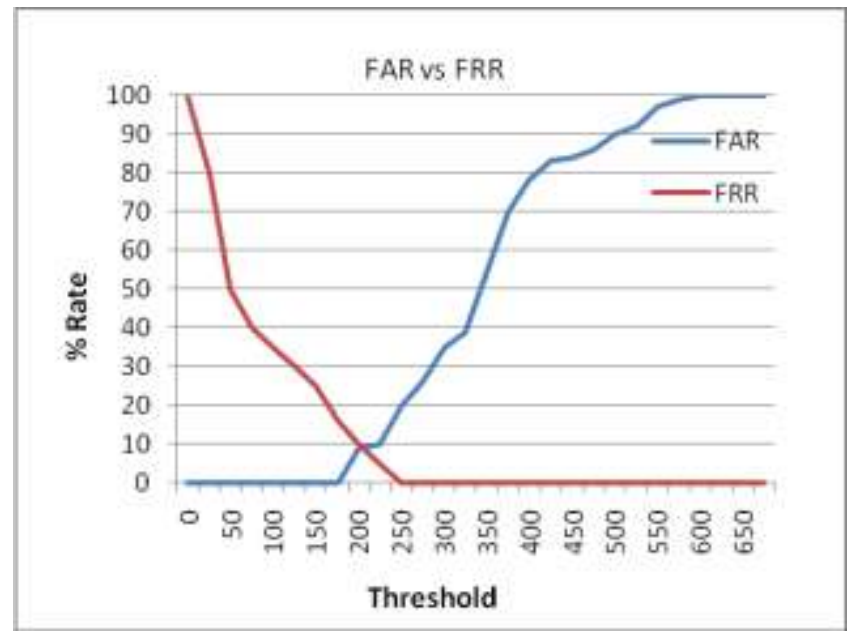

(b)

Figure 7. (a)TAR vs TRR Plot for Gabor Feature without Timestamp (b) FAR vs FRR Plot

\subsection{Gabor Filter based Feature Vector without Timestamp}

We have performed same analysis of the signature verification using Gabor filter based feature vector with timestamp. The performance improved as the feature vectors distance is evaluated by adding the timing information of the signature. The analysis shows that EER for TAR Vs TRR plot is $95 \%$ and the same for the FAR Vs FRR plot is 5\%.

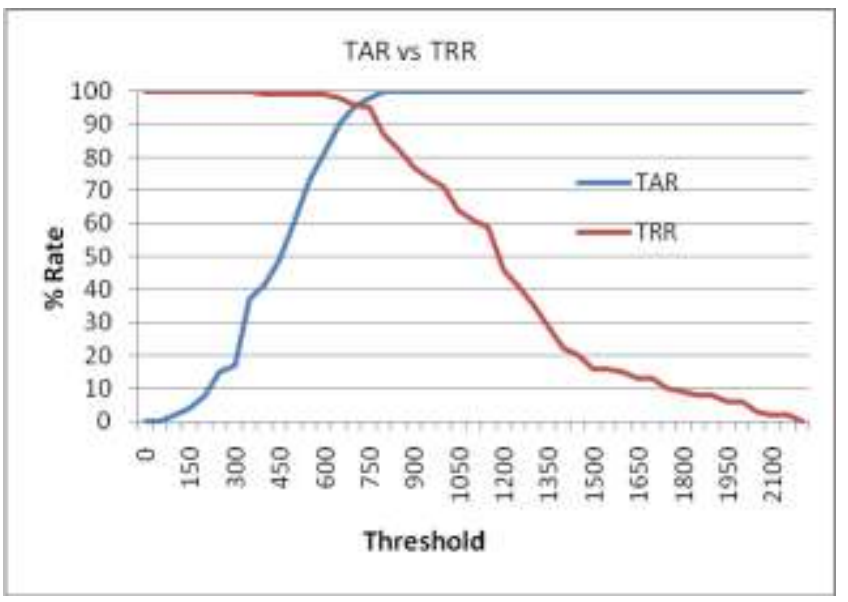

(a)

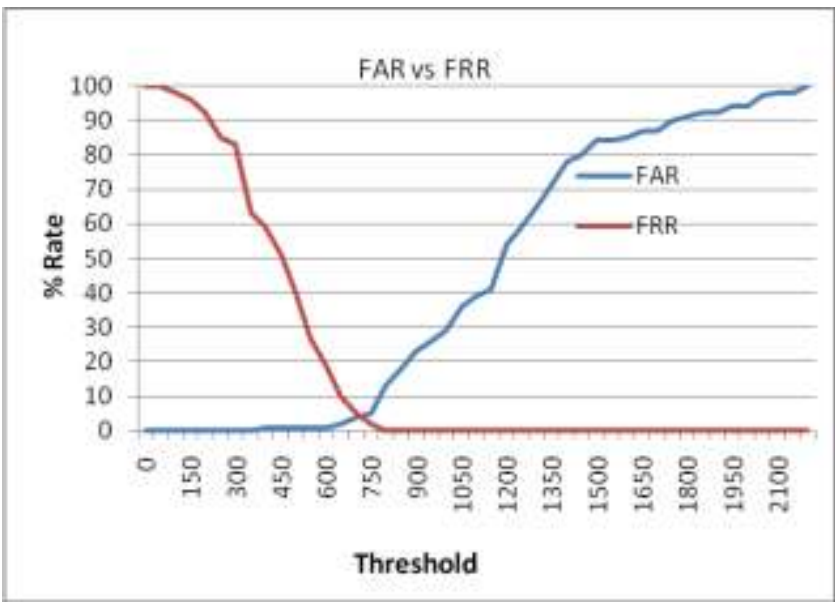

(b)

Figure 8. (a)TAR vs TRR Plot for Gabor Feature with Timestamp (b) FAR vs FRR Plot

\section{CONCLUSION}

In this paper we have proposed a feature vector based on Gabor filtering for the dynamic signature recognition. The algorithm was tested on live signature capturing on Wacom Intuos 4 Digitizer Tablet. The timing information was also incorporated into the Gabor Filter based feature vector. The algorithm gives 90\% EER for TAR-TRR analysis for Gabor feature vector without timestamp. When timing information is added the performance is improved and the reported EER is 95\% for TARTRR analysis. The performance can be further improved by use of good classifier.

\section{REFERENCES}

[1] A. K. Jain, A. Ross, S. Prabhakar, "An Introduction to Biometric Recognition", IEEE Transactions on Circuits and Systems for Video Technology, Vol. 14, No. 1, January 2004

[2] A. K. Jain, A. Ross, and S. Prabhakar, "On Line Signature Verification”, Pattern Recognition, vol. 35, no. 12, Dec 2002. pp. 2963-2972, 
[3] A. Zimmer and L.L. Ling, "A Hybrid On/Off Line Handwritten Signature Verification System", Seventh International Conference on Document Analysis and Recognition, vol.1, pp.424-428, Aug.2003.

[4] D. Hamilton, J. Whelan, A. McLaren, "Low cost dynamic signature verification system", Security and Detection, 1995. IEEE CNF European Convention, 16-18 May 1995. pp 202 206.

[5] R. Plamondon, G. Lorette, "Automatic Signature Verification and Writer Identification - The State of the Art", Pattern Recognition, vol. 4, no. 2, pp. 107-131, 1989.

[6] R. Plamondon, "The design of an On-line signature verification system", Theory to practice, International journal of Pattern Recognition and Artificial Intelligence, (1994). pp 795-811.

[7] H B kekre, V A Bharadi, "Specialized Global Features for Off-line Signature Recognition", 7th Annual National Conference on Biometrics RFID and Emerging Technologies for Automatic Identification, VPM Polytechnic, Thane, January 2009

[8] H B Kekre, V A Bharadi, "Signature Recognition using Cluster Based Global Features", IEEE International Conference (IACC 2009), Thapar University, Patiala- Punjab, India. March 2009,

[9] H. Baltzakis, N. Papamarkos, "A new signature verification technique based on a two-stage neural network classifier", Engineering Applications of Artificial Intelligence 14 (2001)

[10] H. Dullink, B. van Daalen, J. Nijhuis, L. Spaanenburg, H. Zuidhof, "Implementing a DSP Kernel for Online Dynamic Handwritten Signature Verification using the TMS320 DSP Family”, EFRIE, France December 1995 SPRA304.

[11] H. lei, S. Palla and V Govindraju, "ER2: an Intuitive Similarity measure for On-line Signature Verification", Proceedings of CUBS 2005.

[12] T. Rhee, S. Cho, "On line Signature Recognition Using Model Guided Segmentation and Discriminative feature selection for skilled forgeries", IEEE Transaction on pattern recognition, Jan 2001

[13] V. Nalwa, "Automatic On-Line Signature Verification", proceedings of the IEEE Transactions on Biometrics, vol. 85, No. 2, February 1997.

[14] R. Doroz, K. Wrobel "Method of Signature Recognition with the Use of the Mean Differences",
Proceedings of the ITI 2009 31st Int. Conf. on Information Technology Interfaces, June 22-25, 2009.

[15] SVC (Signature Verification Competition) database available at the website http://www.cse.ust.hk/svc2004/index.html

[16] H. B. Kekre, V A Bharadi, "Using Component Object Model for Interfacing Biometrics Sensors to Capture Multidimensional Features", IJJCCT 2009, Shanghai, China, Dec 2009

[17] L. Hong, A.K. Jain, "Fingerprint Image Enhancement : Algorithm and Performance Evaluation", IEEE transaction on Pattern Analysis and Machine Intelligence, Vol. 20, No. 8, August 1998

[18] H B Kekre, V A Bharadi, "Dynamic Signature Preprocessing by Modified Digital Difference Analyzer Algorithm”, ThinkQuest 2010, March 2010, www.springerlink.com

[19] H. B. Kekre, S. Bhatnagar,"Finger Print Matching Techniques", Proceedings of National Conference on Applications Digital Signal Processing. (NCDSP - 2007), Mumbai, Jan 19 - 20, 2007

[20] M. Laadjel, A. Bouridane,F. Kurugollu, S Boussakta, "Palmprint Recognition using Fischer-Gabor Feature Extraction", Proceedings of IEEE International Conference ICASSP 2008, IEEE DOI : 1-4244-1484-9

[21] C. Z. Wen, J.S. Zang, "Palmprint Recognition based on gabor Wavelets and 2-Dimensional PCA", Proceedings of the 2007 International Conference on Wavelet Analysis and Pattern recognition, Beijing, China, IEEE DOI : 1-4244-1066-5/07, 2007

[22] F. Alonso, J.Fierrez, J. Ortega, "An Enhanced Gabor Filter-Based Segmentation Algorithm for Fingerprint recognition Systems", Proceedings of the 4th International Symposium on Image and Signal Processing and Analysis (2005).

[23] H B Kekre, V A Bharadi, "Fingerprint \& Palmprint Segmentation by Automatic Thresholding of Gabor Magnitude", ICETET 2010, Nagpur, India, IEEE CNF

[24] H B Kekre, V A Bharadi , "Using Component Object Model for Interfacing Biometrics Sensors to Capture Multidimensional Features", IJJCCT, Dec 2009, China

[25] A K. Jain, S Prabhakar, L Hong, "A Multichannel Approach to Fingerprint Classification", IEEE Transactions On Pattern Analysis And Machine Intelligence, Vol. 21, No. 4, April 1999 